\title{
The Rotating Discs Argument Defeated
}

\author{
J. Butterfield ${ }^{1}$ \\ All Souls College, Oxford OX1 4AL
}

Tuesday 25 May 2004

\begin{abstract}
The rotating discs argument (RDA) against perdurantism has been mostly discussed by metaphysicians, though the argument of course appeals to ideas from classical mechanics, especially about rotation. In contrast, I assess the RDA from the perspective of the philosophy of physics. I argue for three main conclusions.

The first conclusion is that the RDA can be formulated more strongly than is usually recognized: it is not necessary to "imagine away" the dynamical effects of rotation. The second is that in general relativity, the RDA fails because of frame-dragging.

The third conclusion is that even setting aside general relativity, the strong formulation of the RDA can after all be defeated. Namely, by the perdurantist taking objects in classical mechanics (whether point-particles or continuous bodies) to have only temporally extended, i.e. non-instantaneous, temporal parts: which immediately blocks the RDA. Admittedly, this version of perdurantism defines persistence in a weaker sense of 'definition' than pointilliste versions that aim to define persistence assuming only instantaneous temporal parts. But I argue that temporally extended temporal parts: (i) can do the jobs within the endurantism-perdurantism debate that the perdurantist wants temporal parts to do; and (ii) are supported by both classical and quantum mechanics.

This is an extract from a much longer paper, which is available at: http://philsci-archive.pitt.edu/archive/00001760. The main differences are that the longer paper: (i) gives much more detail about the form and scope of the RDA, the interpretative subtleties of classical mechanics, and the physics of rotation; and (ii) reports and assesses several other replies to the RDA, especially those by Callender, Lewis, Robinson and Sider.
\end{abstract}

\footnotetext{
${ }^{1}$ email: jb56@cus.cam.ac.uk; jeremy.butterfield@all-souls.oxford.ac.uk
} 


\section{Introduction}

This paper is an attempt to connect what modern physics says about matter, with the debate in analytic metaphysics about whether an object persists over time by the selfsame object existing at different times (nowadays called 'endurance'), or by different temporal parts, or stages, existing at different times (called 'perdurance'). Though this is a multi-faceted debate, with various connections to the philosophy of physics, I will focus on just one connection: a metaphysical argument against perdurantism, based on the idea of rotating homogeneous matter, and nowadays often called the 'rotating discs argument' (RDA). I will argue, against much of the literature, that the argument fails, because of some features of classical mechanics (including how it should be interpreted in the light of quantum mechanics).

I begin by reporting the RDA and some of the literature's discussion of it (Section 2 ). Then I propose an improved version of the argument; but point out that even this version fails in general relativity (Section 3). In the last two Sections, I set aside general relativity and propose a new perdurantist line of reply to the argument (even my improved version of it). The idea is that the perdurantist should accept only non-instantaneous temporal parts. This idea can be supported both by considerations drawn from classical mechanics (Section 4), and by considerations drawn from quantum mechanics (Section 5).

This paper is a fragment of a larger project. My $(2004,2004 \mathrm{a})$ describe some other connections between the endurantism-perdurantism debate and aspects of physics and its philosophy. My (2004) also presents the debate in much more detail than I can here. Suffice it to say that I conceive the debate in much the same terms as Sider's and Hawley's fine recent monographs (2001). As to the RDA itself, my (2004b) expands on the material to follow. (Hawley and Sider also both discuss the RDA, at pp. 72-90, and 224-236 (respectively), of their monographs (2001).)

\section{The story so far}

\subsection{The RDA}

The argument envisages that the perdurantist with her ontology of temporal parts faces the project of defining persistence: since persistence is not identity, she needs to tell us what it is. (This project is also called 'analyzing persistence', and 'analyzing or defining the genidentity relation between temporal parts'.) In particular, she needs to define persistence so as to distinguish "ordinary persisting objects" (i.e. the referents of ordinary terms, and elements of ordinary domains of quantification) from the countless other "spacetime worms", i.e. mereological fusions of temporal parts. (Most perdurantists accept unrestricted mereological composition, so that they also accept these worms as genuine objects.) On pain of circularity, the definiens is not to 
presuppose the notion of persistence.

The argument urges that the perdurantist cannot succeed in this project. $^{2}$ It is based on two ideas:

(i) Homogeneous: In a continuum (i.e. a continuous body whose composing matter entirely fills its volume) that is utterly homogeneous throughout a time-interval containing two times $t_{0}, t_{1}$, a spatial part at the time $t_{0}$ is equally qualitatively similar to any spatial part congruent to itself (i.e. of the same size and shape) at the later time $t_{1}$. (The properties of the continuum can change over time, but must not vary over space, even on arbitrarily small length scales; e.g. the continuum could cool down, but must at each time have the same temperature everywhere.)

(ii) Follow: The perdurantist will presumably try to define persistence in terms of suitable relations of qualitative similarity between temporal parts. The obvious tactic is to have the definiens "follow" the curves in spacetime that are timelike and track maximum qualitative similarity.

The tactic of Follow seems to work well when applied to point-particles moving in a void each with a continuous spacetime trajectory (worldline). For however exactly we define 'maximum qualitative similarity', there will no doubt be, starting at a pointparticle at $t_{0}$, a unique timelike curve of qualitative similarity (or occupation-by-matter) passing through it: the worldline of the particle. Similarly for point-particles moving, not in a void, but in a continuous fluid with suitably different properties - a different "colour", or made of different "stuff", than the point-particle. ${ }^{3}$

But Homogeneous implies that Follow's strategy stumbles when applied to an utterly homogeneous continuum. There are altogether too many spatial parts at $t_{1}$ that are tied-first-equal as regards qualitative similarity to the given spatial part at $t_{0}$ : any congruent spatial part will do. In other words: the curves of qualitative similarity run "every which way".

This problem is made vivid by urging that the perdurantist cannot distinguish two possibilities that, the argument alleges, must be distinguished. One main example, on which I will focus, is the case of a rigid disc made of homogeneous matter that is stationary; and a duplicate disc (rigid and congruent to, made of the same homogeneous material as, at the same temperature as etc. the first) that is rotating about the axis through its centre. It will be convenient to have labels for two such possibilities: call them '(Stat)' and '(Rot)'.

Hence the argument is nowadays often called the 'rotating discs argument' (RDA). (In some discussions, both discs are rotating, but with different velocities, maybe even in different senses.) But all agree that countless other examples would serve just as well

\footnotetext{
${ }^{2}$ The RDA arose in recent philosophy in Kripke (unpublished lectures) and Armstrong (1980). Zimmerman (1998) reports how the argument goes back at least to Broad in 1925. Sider (2001, p. 226) notes that Leibniz (1698, sect. 13) deploys essentially the same argument: but Leibniz's target is Descartes' doctrines about matter and motion.

${ }^{3}$ Agreed, one can object to this tactic. I address these objections in Sections 4.1-4.2.1 of my (2004a). But the details of my replies are not needed for this paper: for they are no help to the perdurantist in facing the trouble made by the RDA.
} 
as a disc: e.g. a sphere; or a body of fluid, like a river, that can be either stationary or flowing (or flowing with different speeds, or in different directions).

It seems that the endurantist can easily distinguish the two possibilities, according to whether the very same non-circularly-symmetric part, e.g. a segment, is in the same place at two times. My (2004b) pursues the question whether this is really so: can the endurantist legitimately use the notion of being in the same place at two times, i.e. the notion of persisting spatial points? (This question is almost entirely ignored in the metaphysical literature: authors often appeal without further discussion to the idea of "the same place" (e.g. Hawley 2001, p. 85).) Here I just assume, in order to give the RDA as good a run as possible, that the answer is Yes; though I will say more in Section 3 about what this notion involves.

On the other hand, it seems the perdurantist has a problem. Surely she must say that all the relations (and therefore, all her proffered "suitable relations" for analysing persistence) between two temporal parts of the disc (say, second-long parts at noon and 12.01) are the same - whether the disc is rotating or not? And similarly for temporal parts at the two times of any spatial part of the disc, such as a segment.

The rest of this Section clarifies the scope of this argument, and the kinds of reply the perdurantist can give to it. This will yield a statement of a consensus which is widespread in the literature - and an announcement of how the remainder of the paper will argue against that consensus.

\subsection{Intrinsic properties and velocities}

So far I have expressed the RDA's main idea as the inadequacy, for defining persistence, of qualitative similarity. But in some versions of the argument, the emphasis is instead on the inadequacy of intrinsic properties. The intrinsic-extrinsic distinction among properties is controversial, but the rough idea is that possession of an intrinsic property implies nothing about the possessor's environment, i.e. about matters of fact beyond the instance. So in these versions, the target is a perdurantist who seeks a definiens using intrinsic properties of temporal parts. And in some versions, the target is a yet stronger neo-Humean doctrine to the effect that (roughly speaking) all facts - not just facts of persistence - are determined by all the various intrinsic properties of all the points of spacetime. The most influential version of this sort of extreme pointilliste doctrine is Humean supervenience, as formulated and defended by Lewis (1986, p. ix-x; 1994, p. 474; 1999).

This emphasis on intrinsic properties goes along with an objection to the obvious suggestion that what distinguishes the two cases is the direction of the instantaneous velocity of the disc's (or sphere's, or river's) constituent parts. Thus for the stationary disc, all the disc's parts have zero velocity; while for the rotating disc, the parts have various velocities (and for a perfectly rigid disc, a common angular velocity); and similarly for the sphere or river. But, says the RDA, the notion of velocity presupposes the persistence of the object concerned. For average velocity is a quotient, whose 
numerator must be the distance traversed by the given persisting object: otherwise you could give me a superluminal velocity by dividing the distance between me and the Sun by a time less than eight minutes. So average velocity's limit, instantaneous velocity, surely also presupposes the notion of persistence. Accordingly, says the RDA, the perdurantist cannot adopt the obvious suggestion, of distinguishing the cases in terms of instantaneous velocity (or angular velocity) - on pain of circularity.

The notion of presupposition, like the intrinsic-extrinsic distinction, is controversial. Besides, Tooley (1988) proposes a heterodox account of instantaneous velocity as an intrinsic property of an object at a time - though he is not concerned with the debate over persistence; and Bigelow and Pargetter (1989, 1990 Section 2.6) propose a similar account of velocity. But in this paper, I will set aside these controversies and concede the above objection. That is, I will assume that both average and instantaneous velocity presuppose the notion of persistence, and are extrinsic properties. But I shall nevertheless argue in Section 4 that a certain kind of perdurantist can endorse the obvious suggestion, i.e. can appeal to velocities to distinguish the two cases.

\section{3 "Naturalism"}

So far, my description of the perdurantist project of defining persistence, and of the RDA against it, might well be read within the tradition of conceptual analysis. By this I mean that the perdurantist's definition would be both finite in length, and formulated using everyday concepts. But nowadays, the literature also considers a "naturalized" perdurantist project of

(a): providing only a supervenience basis for persistence (i.e. allowing infinitely long definitions), rather than a finite definition or analysis of it; and-or

(b): appealing to technical notions, and contingent bodies of doctrine, in particular the laws of dynamics. (Some authors combine (b) with use of the Ramsey-Lewis technique for simultaneous functional definition; e.g. Sider (2001, 230-236).)

Accordingly, the RDA is nowadays sometimes formulated as targeting even: (a) the supervenience of persistence on qualitative similarity among, and-or intrinsic properties of, the perdurantist's temporal parts; where (b) such supervenience may even be contingent, say relative to the laws of a dynamical theory.

This situation prompts two comments: the first relates mostly to (a), the second mostly to (b).

(1): Non-reductive perdurantism:- There is also an even more naturalistic conception of perdurantism, which might well avoid the RDA. On this conception, the perdurantist seeks a theory of perdurance and related concepts, that can appeal to scientific technicalities, that can revise rather than describe our concepts - and that does not have to define persistence in terms that do not presuppose it. Of course, analogous "non-reductive" conceptions are nowadays commonplace in the philosophical study of many concepts, such as causation, perception and action. So just as a philosophical theory of causation might decline to define causation (even infinitarily, 
even by Ramsey-Lewis functional definition), a perdurantist might decline to define persistence (even in these liberalized senses), on the grounds that she nevertheless says enough to adequately distinguish "ordinary persisting objects" from other "spacetime worms". I shall return to this modest (because non-reductive) perdurantism in Section 4. But until then I shall consider the more ambitious perdurantist, who aspires to define persistence, and so faces the RDA.

(2): How many worlds?:- Once we allow that a perdurantist theory of persistence might appeal to a contingent body of doctrine, such as a physical theory, the discussion of the RDA (or even the whole endurantism-perdurantism debate) is liable to become relative to a theory. There are two aspects to this; the first leads on to the second.

First: the RDA might hold good in one theory, and fail in another. Thus it is a familiar thought that any consistent theory lays out a set of possibilities: in philosophical jargon, possible worlds according to the theory; in physics jargon, a space of solutions. So relative to any consistent theory about matter and rotation (describing them no doubt partially rather than completely - and perhaps falsely), the two cases (Stat) and (Rot) are either two distinct possibilities: or they are not, either because at least one is not possible (since e.g. the theory denies that matter is homogeneous), or because they are the same possibility.

This point is independent of "scientific realism", which concerns whether we should believe the theoretical claims of our best theory to be at least approximately true. But of course, any "naturalist" will be especially interested in whether the RDA holds good in our best theory of matter and rotation.

Second: But we should beware of just dismissing the RDA on the ground that according to our best theories, matter is in fact made of atoms and so not homogeneous. For presumably:

(i): A continuous, rigid and utterly homogeneous form of matter could exist and be formed into a disc that either rotates or is stationary. And:

(ii): No philosopher of persistence is "so far gone" in naturalism as to be interested only in how objects persist, given all the contingencies of the actual world.

In what follows, I will agree with these presumptions, so as to give the RDA against perdurantism as good a run as possible. But it is worth drawing attention to them since, as we shall see:

(i'): The sort of continuous and homogeneous matter the RDA needs is a much subtler and more problematic affair than the RDA literature typically recognizes. ${ }^{4}$ This leads in to (ii'):-

(ii'): Some perdurantists reply to the RDA by saying that for the possibilities (Stat) and (Rot) to exhibit no difference to which the perdurantist can appeal, the advocate of the RDA needs to "imagine away" so many actual laws, technical and-or everyday, which describe various causes and effects of rotation, that the RDA's possibilities (Stat) and (Rot) are, though logically or metaphysically possible, very arcane. Indeed, they are so arcane that a naturalist perdurantist need feel no shame in being unable to

\footnotetext{
${ }^{4} \mathrm{My} 2004,2004 \mathrm{~b}$ develop this theme.
} 
accommodate them.

To put the reply (ii') in the jargon of possible worlds: the perdurantist claims their theory of persistence, though contingent and unable to discriminate the possibilities (Stat) and (Rot), is true in so broad a class of possible worlds that excluding (at least one of) (Stat) and (Rot) is a small price - and worth paying. (Examples of this reply include: Lewis (1986, p.xiii, 1994, p. 475), Callender (2001), and (less explicitly) Sider (2001, 230-236).) This leads to the next Subsection.

\subsection{The accompaniments of rotation}

Rotation has countless typical causes and effects; or if one is wary of causal talk: countless typical accompaniments. Typically, a rotating object was previously set in motion, say by being pushed by someone, and exhibits distinctive dynamical effects: for example, a solid object tends to become oblate, and a fluid, like water in a whirlpool, develops a concave surface. These accompaniments do not depend on matter being in fact atomistic (or on the laws of physics being relativistic and quantum). So in a possible world that contained continuous and homogeneous matter but was otherwise "like the actual world", these accompaniments - even the "technical" ones, like oblateness and concavity - would occur. In which case, the RDA needs to block the perdurantist appealing to them so as to distinguish the cases.

True to the tradition of conceptual analysis, the literature on the RDA almost entirely sets aside the technical accompaniments, and concentrates on the everyday ones, like having been pushed in the past; and on related everyday counterfactuals, such as 'were I to spray a spot of paint on the disc, I would see it move', or 'were I to grasp the disc, I would feel friction'. More specifically, the literature tends to assume that the RDA can legitimately set aside all the technical accompaniments by just stipulating that the rotating disc is not only solid but perfectly rigid, so that it does not become oblate; (hence Section 2.1's mention of rigidity). The philosophical battle can then be joined on two battlefields familiar to metaphysicians; as follows.

First, there is debate about whether the RDA can legitimately "imagine away" the everyday accompaniments of rotation, so that the perdurantist cannot appeal to them. In particular: if (as usual) the RDA stipulates that the present and "occurrent" everyday accompaniments are absent, can the perdurantist appeal to past or future accompaniments, or perhaps to counterfactuals about them? For example:

(i) Can the perdurantist make the distinction by appealing to a past cause, such as a push, or to a present counterfactual about seeing a paint-spot move?

(ii) Or would appealing to a past cause amount to postulating an unacceptable "temporal action-at-a-distance" (e.g. Robinson 1989 p. 405-406; Hawley 2001 p. 81)?

(iii) And would appealing to a present counterfactual amount to postulating unacceptably "ungrounded" counterfactual truths (Robinson 1989 p. 403; Hawley 2001 p. 74-75)?

Second, there is debate about whether the perdurantist can appeal to differences 
between (Stat) and (Rot) that are distinctively metaphysical (neither everyday nor technical-physical). For example: Can the perdurantist appeal to:

(i) a special (non-Humean) relation of immanent causation between temporal parts that subvenes (or even yields an analysis of) persistence (Armstrong 1980, 1997, pp. 73-74); or

(ii) special vectorial properties that are numerically equal to, yet different from, velocities (Robinson 1989 pp. 406-408, Lewis 1999: incidentally, this idea echoes Leibniz's proposal against Descartes (1698, sect. 13)); or

(iii) non-causal relations between temporal parts that are not supervenient on the intrinsic natures of the parts that are the relata, and yet are not just spatiotemporal relations (Hawley: 1999, p. 63-66; 2001, p. 85-90)?

I do not need to enter either of these battlefields; (fortunately, since they remain well-populated, despite the crossfire!). As to the first, I can set aside the "everyday accompaniments". For I shall argue in Section 3 that the RDA should not just set aside technicalities, in particular the technical accompaniments of rotation; and that in any case, it cannot do so just by stipulating perfect rigidity. As to the second, I can set aside controversial notions like immanent causation etc. (of which I am in any case wary). For I shall argue in Sections 4 and 5 that technicalities from physics suggest another way the perdurantist can reply to the RDA.

\subsection{Two kinds of reply: Against the consensus}

We can sum up "the story so far" in two stages. First, there are two main ways perdurantists can reply (and have replied) to the RDA. They can either:

('Appealing Differences'): argue that there are differences between the discs to which they can appeal; whether everyday (e.g. 'someone pushed it'), technical (e.g. 'it's oblate') or metaphysical (e.g. 'the timelike curves of immanent causation are helical, not straight'); or

('No Difference'): argue that possible worlds in which the discs show no such difference are too arcane to matter: i.e. they do not fall within the scope of their "naturalist" account of persistence.

Of course, these replies can in a sense be combined. For in the back-and-forth of debate, a perdurantist might move from one reply to the other: "well, if you imagine away all of those accompaniments of rotation, I will then reply that there is after all no difference'.

Second: In the literature on the RDA, considerations of metaphysics, and in particular conceptual analysis, tend to dominate. This dominance has led to a widespread consensus on four points: two in support of the RDA, and two against the perdurantist. Namely:

(I): The RDA can legitimately

(a) imagine away the usual accompaniments of rotation: both the everyday ones; and the technical ones such as discs tending to be become oblate (in the latter 
case, by requiring the discs to be rigid);

(b) assume the intuitive notion of rotation, with its idea of persisting spatial points.

On the other hand:

(II): the perdurantist cannot legitimately can they

(c) appeal to differences of velocity, since velocity presupposes persistence; nor

(d) appeal to the atomic, indeed quantum-theoretic, nature of matter, since the topic of debate is our common-sense conception of persistence-which surely allows continuous matter.

Turning to this paper: I shall now argue against the consensus (a)-(d). Section 3 argues against (a) and (b); Sections 4 and 5 against (c) and (d). The overall effect will be twofold. As to (a) and (b): I will concede that there are sound versions of the RDA. Indeed, the RDA can be formulated more strongly than in Section 2.1: for it need not imagine away the usual accompaniments of rotation. But as to (c) and (d): a certain sort of perdurantist-roughly speaking, one who accepts only noninstantaneous temporal parts - can both appeal to differences of velocity, and garner support for their position from quantum theory.

\section{Describing rotation}

The main theme of this Section is the practice by the RDA's advocates, of imagining away the technical accompaniments of rotation (especially dynamical effects, like oblateness) that the perdurantist might latch on to as marking the distinction between the discs, (Stat) and (Rot). I will argue that:

(i): One main aspect of physics' description of rotation supports this practice (Section 3.1).

(ii): But the RDA's advocates are lucky - it is an undeserved victory - since they are largely unaware of (i); and their actual reasons for imagining away such accompaniments are worse (Section 3.2).

(iii): In any case, the RDA can be formulated more strongly, i.e. without imagining away such accompaniments. The idea is that the perdurantist cannot distinguish two possibilities that differ about the sense of a disc's rotation; (Section 3.3).

(iv): Both the original version of the RDA and this stronger version fail in general relativity (Section 3.4).

\subsection{Rotation is kinematic}

The description of motion and in particular of rotation, in the various spacetime theories (non-relativistic and relativistic, special and general), is a large and subtle subject. In particular, general relativity holds considerable surprises (Malament 2002, 2003). 
But in this Subsection, I only need the following points:

(i): The various theories analyse motion using a four-dimensional connection (not necessarily supplied by an absolute space), and spatial and temporal metrical structure; and they model continuous matter with a congruence of timelike curves: i.e. a continuously infinite collection of worldlines whose points of intersection with a (possibly finite) spacelike slice completely fill the slice.

(ii): Given such a connection, metric(s) and congruence, the theories define (in much the same way) at each point in the congruence a rotation tensor, usually symbolized as $\omega$, which gives a quantitative measure of the speed and direction of rotation of the congruence in an arbitrarily small neighbourhood of that point.

(iii): This construction is a "robust local" limit of definitions of rotation for extended objects; in the following sense. There are various intuitively compelling (and experimentally realizable) criteria for whether an extended object, such as a disc, is rotating; but as one considers smaller and smaller discs, the verdicts of these various criteria as to whether a given disc is rotating converge on the verdict given by the rotation tensor, i.e. by whether or not $\omega=\mathbf{0}$. (The surprises about rotation in general relativity (Malament ibid.) concern extended objects. For more details about (i)-(iii), cf. e.g. Misner et al. 1973, p. 566; Dixon 1978, p. 121-128, 140-145, 163-166; Wald 1984, p. 216-218.)

There is also a large and subtle philosophical subject hereabouts: can the perdurantist and endurantist equally well accommodate the contents of these spacetime theories, or does one have an advantage over the other? Thus the rise of relativity theory prompted philosophers such as Russell and Whitehead to claim that relativity theory favoured an ontology of events, not substances - corresponding, at least roughly, to today's perdurantist ontology. But on the other hand: nowadays, many endurantists are "scientific realists", and even "substantivalists" about spacetime so that they advocate a mixed ontology: though matter endures, spacetime regions are also genuine objects and they perdure. For the sake of brevity, I must set aside this debate: (for some discussion and references, for both sides of this debate, cf. Sider (2001, pp. 75-76, 110-119) and my 2004b.) As mentioned at the end of Section 2.1, I will just assume, so as to give the RDA as good a run as possible, that the endurantist has at least as much right as the perdurantist to these theories.

Points (i)-(iii) imply that $\omega$ is definable in kinematic terms, without regard to dynamics: rotation makes sense without reference to forces, energies etc. I claim this supports the RDA's tradition of imagining away rotation's usual accompaniments. To put it in terms of possible worlds: (i)-(iii) suggest there are possible worlds with a spacetime manifold, spatial and temporal metrics and compatible connection, and a congruence of timelike curves representing continuous matter - and no dynamics. A pair of these worlds can match in countless ways and yet differ as to whether the matter is rotating at a point in spacetime. For we can imagine that the worlds match so well as to justify talking of a point common to the two spacetimes, and yet in one world the rotation tensor is zero at the point, while in the other it is non-zero. I take this as evidence that perdurantism should strive to accommodate the distinction between 
these possibilities. ${ }^{5}$

\subsection{Beware of rigidity}

But advocates of the RDA have usually had different-and I submit, worse-reasons than that just given, for insisting that the perdurantist should distinguish (Stat) and (Rot) even without any of the usual accompaniments of rotation. I will not try to catalogue people's errors, but will focus on one prevalent reason: that the RDA can just stipulate that the discs are perfectly rigid. (Parts of this Subsection's critique will carry over to versions of the RDA that use a homogeneous fluid, rather than a rigid solid.)

This reason is defective in two ways. First: to say these two words 'perfectly rigid', so "trippingly off the tongue", is to forget that within the theories of classical continuum physics, perfect rigidity is a very strong idealization - it violates central principles of these theories.

To take the example of a disc: what in fact would happen when a (classical, continuous, homogeneous) stationary disc is pushed at its edge to make it rotate, is very complicated. A disturbance would travel outward (at the speed of sound for the disc's material) from the place where the push is applied, leading to a complex process that settled down so that the whole disc rotated approximately uniformly, with internal cohesive forces exerting the required centripetal forces on parts of the disc. ${ }^{6}$ So assuming perfect rigidity requires that the disc's cohesive forces should respond "infinitely quickly" to distorting influences. More precisely, it amounts to vetoing any account of how the whole disc is set in motion as a consequence of the motions of the parts. In physics jargon: it vetoes any constitutive theory. ${ }^{7}$

Second: it is not true that perfect rigidity gets rid of all the actual technical accompaniments of rotation. For not all such accompaniments are kinematically manifested, i.e. associated with changes in shape or size. In particular, dynamical effects involving forces and energies would be present in a perfectly rigid rotating disc. (In physics

\footnotetext{
${ }^{5}$ I do not claim that it is conclusive evidence. Some perdurantists will still prefer the 'No Difference' reply to the RDA. That is: they will say that worlds with no dynamics are so unlike the actual world, that perdurantists have no responsibility to distinguish rotation and non-rotation within them (cf. Callender 2001, p. 38). But I do not need to resolve this dispute between myself and fellowperdurantists. For all perdurantists can agree to the more important conclusions in the next three Subsections.

${ }^{6}$ In the actual quantum world, this description is of course an excellent approximation for solid discs, the cohesive forces being electromagnetic forces between atoms.

${ }^{7}$ Two incidental remarks about rigidity. (1): There is also the worry that perfect rigidity violates relativity's prohibition on faster-than-light signals. But in fact, relativistic theories allow generalized notions of rigidity: for a philosopher's introduction, cf. Earman (1989, Chapter 5.5, pp. 98-101). (2): Among Bigelow and Pargetter's arguments for their heterodox account of instantaneous velocity, as not always a limit of average velocities, is a thought-experiment involving perfectly rigid spheres (1989, pp. 292-293, 1990, pp. 67-68). As it happens, I disagree with their argument, but I will not go into details: in Section 2.2, I already set aside such heterodox accounts of velocity.
} 
jargon: some effects involve stress rather than strain.) There will be cohesive forces throughout the disc's interior which would be absent if the disc were stationary; and the disc's energy is greater (which in relativity means that its mass is greater). Though such effects are more technical than being oblate, that is no reason to think the perdurantist cannot appeal to them. ${ }^{8}$

\subsection{An improved RDA: allowing the actual accompaniments}

The previous Subsection prompts the suspicion that the RDA needs to imagine away all the actual accompaniments of rotation, in something like the way suggested in Section 3.1. But in fact, not so! Just by altering appropriately the possibilities considered, we can get a formulation of the RDA which keeps all the actual accompaniments. ${ }^{9}$ Thus the endurantist challenges the perdurantist to distinguish the possibilities:-

(Same): Two perfectly circular discs, $d_{1}$ and $d_{2}$, both made of continuous homogeneous matter and lying in the same spatial plane-but otherwise as different as you please from one another - spin in the same sense (i.e. both clockwise as seen from one side of the plane, and so anti-clockwise as seen from the other side).

(Different): Two discs, $d_{1}^{\prime}$ and $d_{2}^{\prime}$, match $d_{1}$ and $d_{2}$ respectively in all respects (at all times); except that $d_{1}^{\prime}$ and $d_{2}^{\prime}$ spin in opposite senses relative to one another.

The idea is that all the usual accompaniments (stress as well as strain: forces and energies as well as distortion) match between $d_{1}$ and $d_{1}^{\prime}$; and similarly between $d_{2}$ and $d_{2}^{\prime}$. So there is no need to imagine them away.

Nor is there any need for discs within one of the possible worlds to match in any respect, except being perfectly circular, made of continuous homogeneous matter, lying in the same spatial plane - and for (Same), spinning with the same sense.

Three comments, in descending order of importance, by way of clarifying this formulation of the RDA:-

(i): Intuitively, (Different) describes equally well two distinct possibilities: one in which $d_{1}^{\prime}$ spins in the same sense as both $d_{1}$ and $d_{2}$; and the other in which instead, $d_{2}^{\prime}$ shares their common sense of rotation. This contrast of course depends on there being a fiducial spatial direction in common between the possibilities. I agree that this idea is perfectly coherent. But I emphasise that the direction needs to be specified by something salient in the environment, such as a local gravitational field giving one an

\footnotetext{
${ }^{8}$ At least: it is only a reason if we take the endurantism-perdurantism debate as entirely a matter of analyzing everyday concepts. In particular, the RDA cannot just consider an oblate rotating disc and a non-rotating one moulded so as to be congruent to it (as proposed by Hawley 2001, p. 83-84).

${ }^{9}$ Paul Mainwood and David Wallace devised the following formulation in a seminar in autumn 2003. The idea of exploiting the distinction between two senses of rotation, so as to avoid having to imagine away the usual accompaniments, had already been briefly advocated by Dean Zimmerman (1998, p. 268-269), crediting an anonymous referee. But beware: Zimmerman's discussion can be read as placing each disc in a separate possible world - in which case it fails, as explained in (ii) below. Zimmerman kindly points out (personal communication) that this was not his intention; so that his formulation of the RDA is essentially the same as that invented by Mainwood and Wallace. For novelty and precision, I present theirs. My thanks to them and Zimmerman.
} 
up-down distinction, on pain of its being a distinction without a difference, i.e. a spurious distinction - an artefact of a diagram, or of our visual imagination. So: given such a specification, (Different) indeed represents two possibilities. No matter: to challenge the perdurantist, the RDA can simply consider either one of them.

(ii): (This follows on from (i).) The danger of making a distinction without a difference also crops up in another way. As mentioned in footnote 9 , a formulation of the RDA in terms of distinguishing two senses of rotation (and thereby keeping the usual accompaniments) has been urged before, by Zimmerman (1998, p. 268-9). But Zimmerman's brief discussion can be read as challenging the perdurantist to distinguish between a disc rotating clockwise, alone in its possible world, and a duplicate disc rotating counterclockwise at the same rate, alone in its world. And this formulation fails for the reason emphasised in (i): the clockwise-counterclockwise distinction assumes a fiducial spatial direction in common between the possibilities, which for these "lonely" discs is a spurious distinction. (Callender (2001, p. 32, 36-7) seems to read, and object to, Zimmerman in this way.) Our formulation above avoids this difficulty by considering two discs in each possible world, so that we need only intra-world comparisons of the sense of rotation.

(iii): The possibilities can be modified in various ways. In particular, to secure the needed intra-world comparisons of sense of rotation, we do not need two discs. (Same) could instead contain just one disc, rotating in the same sense as a curved arrow drawn on a sheet of paper lying beside it; (Different) would then similarly contain a single disc rotating contrary to the sense of another curved arrow drawn on an adjacent sheet of paper.

\subsection{The RDA fails in general relativity}

But in general relativity, the trajectory of a test-particle falling towards a massive body depends on whether (and how) the body is rotating: the rotating mass "drags", albeit very slightly, the inertial frames in its vicinity (Misner et al. 1973 pp. 699, 879, 1117). This frame-dragging means that the RDA fails in the sense that, in the usual version, the inertial frames (the worldlines of test particles) are dragged around the rotating disc (Rot), but not around (Stat); and in Section 3.3's version, there cannot be the perfect match in rotation's accompaniments both between $d_{1}$ and $d_{1}^{\prime}$ and between $d_{2}$ and $d_{2}^{\prime}$, since the dragging of inertial frames around a rotating body is different, for different senses of rotation. In short: the RDA fails because there is a dynamical effect of rotation, to which a "sufficiently naturalist" perdurantist can appeal so as to answer the challenge of distinguishing the possibilities. ${ }^{10}$

Could the endurantist improve the thought-experiment so as to allow for framedragging, in the kind of way that (Different) and (Same) improve on (Stat) and (Rot) by allowing for the usual accompaniments of rotation? Perhaps, but I do not see how.

\footnotetext{
${ }^{10}$ This argument against the RDA, in its usual version, is due to Callender (2001, p. 38); it is part of his 'No Difference' reply.
} 
On the other hand, the endurantist has two lines of reply, even if she cannot thus improve the thought-experiment. Both return us to some questions raised before.

First, she might emphasise that in developing the RDA for general relativity (in the usual, or Section 3.3's, version) she can stipulate that the discs are "lonely", i.e. that there are to be no test-particles travelling the dragged worldlines. Does this stipulation make the difference to which the perdurantist appeals - viz. whether the frames are dragged, and if so, how - counterfactual? The answer depends on the interpretation of general relativity. Roughly speaking, a substantivalist will answer 'No', since they take the metrical structure of spacetime to be real and occurrent: it is not just an encoding of how suitable bodies would behave. But the endurantist may argue that she can accept general relativity, and so develop the RDA for it, without being a substantivalist in this sense; (cf. Section 3.1). On the other hand, even if we accept that the difference is counterfactual, perhaps the perdurantist can still appeal to it: (cf. Section 2.4).

The second reply is the obvious one about philosophical method. Surely no philosophical account of persistence should be "so far gone" in naturalism as to depend on general relativity: it should be able to accommodate continuous matter in classical and special relativistic spacetimes (cf. (2)(ii) in Section 2.3). And for these cases, the RDA remains unrefuted, at least in Section 3.3's improved version.

I think the second reply has force. But in the next two Sections, I will argue that the perdurantist can meet the challenge of defeating the RDA even outside general relativity: in short, by accepting only non-instantaneous temporal parts. Besides, this version of perdurantism is supported by some heterodox proposals about the intrinsicextrinsic distinction among properties: proposals which are themselves supported by some features of classical and quantum physics.

\section{Perdurantism without tears: the classical case}

\subsection{Rejecting instantaneous temporal parts}

At the end of Section 2.2, I said I would take it that both average and instantaneous velocity presuppose the notion of persistence, and are extrinsic properties. But when we consider a non-instantaneous temporal part, the second point needs to be qualified.

For one of the part's constituent pieces of matter having a certain worldline segment within the part is surely an intrinsic property of the part. And similarly for lesser, i.e. logically weaker, information than the entire worldline segment. For example, that a constituent piece of matter has a certain average velocity over a time-interval "within" the temporal part is intrinsic to the part: notwithstanding the fact that average velocity presupposes the notion of persistence. Similarly for instantaneous velocity at a time "within" the temporal part.

At least, these properties are intrinsic to the part, modulo the topic I set aside at the end of Section 2.1, viz. how to justify the appeal to persisting spatial points, and a spatial metric, that is needed for the idea of the distance traversed by the persisting 
object.

This situation prompts some terminology. Since in this Section (and the next one), I will be focussing on whether the possession of a property $P$ by an object $o$ at a time implies propositions concerning matters of fact, especially about $o$, at other times, it will be convenient to use the phrase 'temporally intrinsic property'. By this I mean "intrinsic as regards time": i.e. roughly, a property whose possession by $o$ at a time implies nothing about matters of fact (especially about $o$ ) at other times (though it may imply propositions about other places). Similarly, I shall talk of temporally extrinsic properties; and of spatially intrinsic and extrinsic properties.

Two warnings about this terminology. (1): I agree that my explanation is vague, not least because the general intrinsic-extrinsic distinction on which it rides is itself vague; (indeed probably ambiguous - cf. Humberstone 1996, Weatherson 2002). But my vague explanation will be enough for this paper. (2): Note that a property could be temporally extrinsic for one instance and not for another. Velocity itself provides an example: it is temporally extrinsic for an object $o$ at an instant $t$; but, as just emphasised, temporally intrinsic to a non-instantaneous temporal part (say, a stage of just $o$ 's "career") that includes $o$ at $t$. Humberstone (1996, p. 206, 227) notes that a similar phenomenon - extrinsic for one instance, but intrinsic for another-occurs for extrinsicality and intrinsicality simpliciter. ${ }^{11}$

These points are of course independent of whether matter is atomic or continuous. The piece of matter can be a point-particle or a point-sized bit of matter in a continuum. (Indeed, the qualification could be stated in the very same words for an extended piece of matter, provided it was small enough for us to model it as point-like, i.e. having a worldline, and a single velocity: but I can focus on unextended pieces of matter.)

To sum up: a non-instantaneous temporal part has a rich set of intrinsic, or at least temporally intrinsic, properties concerning the worldline-segments and average and instantaneous velocities, during the part, of its constituent pieces of matter.

Now consider a version of perdurantism that accepts only non-instantaneous temporal parts. (I will not discuss the pre-history of this proposal in authors like Whitehead: for details cf. Grattan-Guinness (2002). But I will soon discuss whether it should accept all such parts, i.e. parts with an arbitrarily short, though non-zero, temporal extent.)

Since such parts have a rich set of intrinsic properties, the prospects for the perdurantist project of defining persistence (or providing a supervenience-basis for it, or at least some non-reductive account of it) look a great deal better than for a pointilliste version of perdurantism accepting only instantaneous parts (or accepting also extended parts, yet requiring persistence to supervene on the intrinsic properties of instantaneous parts, as in Lewis' Humean supervenience). For with these rich sets of properties, there are so many more ingredients which one could use in the definiens of persistence (or more generally, in the account of persistence). More precisely: the perdurantist's prospects are a great deal better, provided their definition or account

\footnotetext{
${ }^{11} \mathrm{My}(2004,2004 \mathrm{~b})$ further discuss temporal and spatial extrinsicality.
} 
of persistence can legitimately refer to these intrinsic properties of non-instantaneous temporal parts.

In the rest of this paper, I will endorse this version of perdurantism, both in general and as a reply to the RDA (both the usual formulation and Section 3.3's stronger one).

The reply it affords to the RDA is as follows. The worldline segments, average velocities and instantaneous velocities of point-sized bits of matter within a homogeneous disc provide intrinsic properties of the disc's temporal parts. Assuming that the perdurantist can appeal to these intrinsic properties - an assumption I will discuss in Section 4.2 - she can certainly distinguish the discs. Indeed, with these intrinsic properties to hand, she may well have no more of a problem about her project of defining persistence, for the parts of a perfectly circular homogeneous disc, than for the parts of an inhomogeneous one. There are two aspects to this, which we can call 'kinematical' and 'dynamical'.

4.1.1 "Kinematics" First, the perdurantist can appeal to the mathematical fact that every suitably smooth vector field $U$ defined on a open region $R$ of spacetime has integral curves throughout $R$ : curves which are timelike, by definition, if $U$ is timelike. ('Suitably smooth' requires only that $U$ be $C^{1}$, i.e. the partial derivatives of its components exist and are continuous.) So the idea is that the intrinsic properties of a non-instantaneous temporal part of a classical continuous body (whether homogeneous or not) specify the vector field $U$, of instantaneous velocities (to be precise: 4-velocities) of the body's point-sized bits of matter, on the spacetime region $R$ of the part. $U$ then specifies integral curves, i.e. the worldlines within $R$ of the bits of matter. Besides, by considering a set of such non-instantaneous parts that "cover" the entire period for which a given bit of matter exists, its entire worldline can be reconstructed.

There are two points to make about this proposal; of which the second will lead us to "dynamics".

(1): Agreed, this proposal seems at first sight a cheat, a case of theft over honest toil. But I am for the moment just assuming that the perdurantist can appeal to intrinsic properties of non-instantaneous parts, even though some of them involve the notion of persistence: postponing discussion to Section 4.2. And rest assured, I will there admit that this assumption makes this kind of perdurantism "non-reductive".

(2): The idea of reconstructing an object's entire worldline by concatenating segments (each lying in one of a "covering" set of non-instantaneous temporal parts) is closely related to a formal equivalence between the ways that endurantism and perdurantism describe the motions of both point-particles and continuous bodies (in both non-relativistic and relativistic spacetimes). I develop this equivalence in Butterfield (2004, 2004a). The idea, for the simplest case viz. point-particles or point-sized bits of matter in a continuous body, is that:

(a): an endurantist will represent the motion by a single function $q: t \mapsto q(t) \in$ $\mathcal{M}$, mapping times at which the point-particle, or point-sized bit of matter in a continuous body, exists, to locations in a manifold $\mathcal{M}$ (either space or spacetime); while 
(b): the perdurantist will use a collection of functions, labelled by time-intervals that together cover the object's lifetime; for example, if it exists throughout the closed time-interval $[a, b]$, there might be a function $q_{[a, b]}: t \in[a, b] \mapsto q_{[a, b]}(t) \in \mathcal{M}$.

In this paper, I do not need more details of this equivalence (e.g. about extending it to spatially extended objects). It suffices to say that one can reconstruct worldlines from such functions, even for a point-sized bit of matter in an utterly homogeneous continuum, provided the functions' domains are non-degenerate time-intervals, i.e. not singleton sets of times; (cf. Butterfield 2004a, Section 3).

This reconstruction of worldlines from a collection of functions raises two points. First, I admit again that it seems at first sight a case of theft over honest toil: the perdurantist reconstructs worldlines from functions that involve the notion of persistence. Here I again refer to Section 4.2's discussion.

Second, this reconstruction of worldlines is "kinematical". It uses no information about the properties of the moving matter, in particular the causes of its motion ("dynamics"): it simply invokes a set of functions that immediately specify worldlinesegments. So it is natural to ask whether our kind of perdurantist can give an account of persistence that in some way appeals to (i) the properties of the moving matter, or (ii) the causes of its motion.

The RDA argues that appealing to (i) cannot work for perfectly homogeneous matter, though (i) is of course the bread-and-butter of the philosophical search for criteria of identity for actual objects of various sorts, such as persons. I believe that nothing in our perdurantist's rejection of instantaneous temporal parts undermines her appealing to (i) for that kind of search; I will support this in Section 4.2.2 (cf. also Butterfield 2004b, Section 6.2.2.(2)). On the other hand, (ii) leads to "dynamics". ${ }^{12}$

4.1.2 "Dynamics" Our perdurantist can indeed appeal to dynamics. That is: if she is sufficiently "naturalist" that she is willing to appeal to the laws of motion (Section 2.3), then in a classical mechanical world, the definition of persistence can "piggy-back" on the determinism of those laws.

That is: in common cases, the classical laws (above all, Newton's second law, that Force $=$ mass $\times$ acceleration) fully determine the motion of a point-particle, or a pointsized bit of matter in a continuum, over an interval of time $\left[t_{1}, t_{2}\right]$, in terms of its initial position and velocity at $t_{1}$ and the regime of forces on it during $\left[t_{1}, t_{2}\right]$ : all of which the perdurantist can take to be given by intrinsic properties of a temporal part that begins before $t_{1}$ and ends after $t_{2}$.

Agreed, that is rough speaking: hence my 'in common cases'. For accuracy, I should note some of the subtleties, in particular the threat to determinism from solutions in which some quantities become infinite within a finite period of time after the initial

\footnotetext{
${ }^{12}$ This "kinematics-dynamics" contrast exemplifies two more general contrasts in the philosophy of identity (discussed in in my 2004a, Section 4.1) which I call (a) 'ontic-epistemic' and (b) 'conceptualempirical'. (a) concerns whether the criterion or account of identity specifies the "constitutive facts" of persistence, or our grounds-everyday or technical, occasional or systematic - for judgments of persistence. (b) concerns whether the criterion or account eschews the concepts and results of empirical theories, e.g. physical theories, or is willing to invoke them.
} 
time $t_{1}$. For point-particles, such solutions are known to exist even if we veto collisions; for a popular account of this, cf. Diacu and Holmes (1996, Chapter 3). For continua, whether there are such solutions is a deep open question: witness the fact that one of the Clay Institute's million-dollar Millennium Prizes is for a proof or disproof of the rigorous existence for all times of solutions of the equations that govern a classical fluid, i.e. the Navier-Stokes equations. ${ }^{13}$

But for present purposes, I can discount these subtleties: here it is enough to suggest that a naturalist perdurantist can go about defining persistence in terms of integrating the equations of motion.

So much by way of replying to the RDA. But I need to defend this version of perdurantism, especially the assumption that the perdurantist's account of persistence can appeal to the non-instantaneous parts' intrinsic properties. I will defend this perdurantism in four stages. The first two stages are metaphysical; I expound them in the next two Subsections. The third and fourth stages will return us to the philosophy of physics. The third stage, in Section 4.4, concerns the classical mechanical description of motion; the fourth stage, in Section 5, concerns quantum theory. The third and fourth stages will also each involve a novel proposal about the intrinsic-extrinsic distinction among properties.

\subsection{Intrinsic properties of non-instantaneous temporal parts}

Intrinsic properties of non-instantaneous temporal parts raise three issues; which I address in three Subsections.

4.2.1 Can the perdurantist appeal to them? I claim that the perdurantist can legitimately appeal to these parts' intrinsic properties, even though some of them involve the notion of persistence. Does this mean that my sort of perdurantist just gives up on the project of defining persistence (or at least providing a supervenience basis for it) in terms that do not presuppose it? Agreed, giving up need not spell defeat for perdurantism. For a non-reductive perdurantism of the sort mentioned in (1) of Section 2.3 might have various merits - and merits that are not undermined by accepting only non-instantaneous temporal parts. (I will support this in Section 4.3.) But does my sort of perdurantist give up?

Yes and No! Yes, in that she aims to give some account of persistence, yet is willing to have the account invoke notions that presuppose persistence; in particular, instantaneous velocity.

But also, No: for reasons hinted at in Section 4.1.B's discussion of persistence "piggy-backing" on the laws of motion. That is: my sort of perdurantist need not assume persistence as a primitive — or that persistence is somehow satisfactorily defined

\footnotetext{
${ }^{13}$ For a popular account, cf. Devlin (2002, Chapter 4); for a monograph discussion of what is known about the simpler case of a perfect fluid (Euler's equations), cf. Section 4.4 and Example 5.5.8 of Abraham and Marsden (1978) — thanks to Gordon Belot for this reference.
} 
(or accounted for, say with a supervenience thesis) - for some specific set of parts: say, a set that covers the lifetime of the persisting object in question, or a set containing all those temporal parts with a temporal extent (lifetime) less than some bound. She can perfectly well pursue the project of defining, or accounting for, persistence as a relation between any two non-instantaneous parts (including any two sub-parts of any given non-instantaneous part).

And even if the perdurantist accepts all such parts, so that there are parts with arbitrarily short, though non-zero, temporal extents, I maintain that this need not involve a vicious regress of endlessly deferred definitions or accounts of persistence. For the account may, for time-intervals less than some amount, become suitably "uniform", i.e. with no substantive variations for shorter times. In short: it can be "turtles all the way down", provided that below a certain level, the turtles are all the same. Of course, this is in effect what happens in an account of persistence that piggy-backs on the classical deterministic laws of motion, determining future and past positions in terms of present position and instantaneous velocity (or momentum).

4.2.2 Temporal intrinsicality at an instant is rare I turn to a general point about the sorts of property invoked in an account of persistence: a point that applies to both endurantist and perdurantist, and to accounts of criteria of identity for specific kinds of object, e.g. persons, where there are issues, e.g. about the weighing of diverse factors such as bodily and psychological similarity, absent from the highly general endurantism-perdurantism debate.

The point is simply that almost no properties are temporally intrinsic to their instance at an instant. That is: almost all properties require features of their instance not only at a single instant, but also at other (albeit perhaps close) times. So an account of persistence, or a criterion of identity for a specific kind of object, needs must appeal to temporally extrinsic properties; (though the other times involved may be close to the given one).

Unfortunately, this fact is obscured in most philosophical discussion of persistence (at least in the tradition of conceptual analysis). This discussion focusses on the idea of giving an account of, or criterion for, $o$ at time $t$ being the same persisting object (maybe of a specific kind, e.g. person) as $o^{\prime}$ at $t^{\prime}$, that invokes everyday properties. Almost always, the idea is that the object(s) (in perdurantist terms: the two temporal parts) need to be:

(i) suitably similar as to these properties: where 'suitably similar' allows considerable change provided there is some kind of chain of small changes; (cf. Follow in Section 2.1); and-or

(ii) suitably causally related, with the properties being the causally relevant ones (in other jargon: part of the specification of the object's causal state); where again there can be a suitable chain of stages or states linked by causation.

So far, so good: I have no objection to searching for this sort of account or criterion, nor to its invoking everyday properties in ways (i) and-or (ii). But the locution 'at time $t$ ', and the focus on everyday properties, makes philosophers often choose as their 
examples observational properties, i.e. properties which can be ascribed "at a glance": be they "everyday-taxonomic" like 'is a rock/leaf/chair' or "purely sensory" like "is red/hot'. And since they can be ascribed at a glance, philosophers are tempted to think they are temporally intrinsic in the strong sense of requiring something of their instance only for a instant. ${ }^{14}$

And that is false. We are very gross creatures: our perceptual apparatus is insensitive to such properties. Rather, the process of perception "averages", in myriadly complex (and often adaptive) ways, over the instant-by-instant properties of not only the object but also the medium, and our perceptual apparatus itself. So any observational property is temporally extrinsic at an instant: it demands features of its instance over a time-interval of at least about one twentieth of a second - and in general a very complex, open-ended and vague array of features, to boot.

When we set aside conceptual analysis and everyday properties, and consider the properties of technical science, in particular physical theories, the same conclusion holds good: most properties are temporally extrinsic at an instant (though as emphasised, they may well be intrinsic to a non-instantaneous temporal part). Thus most of the hundred-odd physical quantities that get an entry in a physics dictionary are clearly temporally extrinsic at an instant. I have already mentioned velocity: obviously momentum, angular momentum and kinetic energy are temporally extrinsic for the same reason. Many other quantities, such as temperature, conductivity (thermal and electrical), permeability and permittivity, depend for their definition (as well as their value) on collective phenomena that require a process or situation to last longer than an instant (though perhaps much less than a second).

I admit that within classical physics, three familiar quantities are good candidates for being temporally intrinsic even to an instant: viz. position, mass and electric charge. Besides, for a point-particle: these also seem to be spatially intrinsic at a spatial point, not just for an extended spatial region. At least, this is so modulo the topic I set aside at the end of Section 2.1, about the basis of spatial geometry: that is to say, a "relationist" about spatial geometry would no doubt object to the claim that the position of a point-particle is spatially intrinsic to a point. ${ }^{15}$

I also admit that this trio seeming to be intrinsic - taken together with the great success of classical physics in reducing much of the behaviour of large complex objects to the classical mechanics and electrodynamics of postulated tiny components, whether point-particles or point-sized bits of matter in a continuum ("micro-reductionism") has undoubtedly been one strong reason, perhaps the main reason, for the prevalence

\footnotetext{
${ }^{14}$ All parties can agree that among non-observational everyday properties, most are temporally extrinsic; indeed they often require features at other times of objects other than their instance: for example, being married requires a spouse at a past wedding, and no intervening divorce or death.

${ }^{15}$ Beware! For a point-sized bit of matter in a continuum, the trio of position, mass-density and charge-density seem to be not only temporally intrinsic, but also spatially intrinsic - provided we can interpret the densities (i) as defining mass and charge through integration, rather than (ii) being themselves defined from the masses and charges of finite volumes, by taking the limit of smaller and smaller volumes. But in fact, we cannot interpret the densities like this: (i) fails, and we need (ii) — another mark against pointillisme, in my view (2004).
} 
in philosophy of pointilliste doctrines like Lewis' Humean supervenience.

Of course, the RDA is precisely an argument that such doctrines come to grief on the topic of persistence. ${ }^{16}$ And my present point is that the rarity of temporal intrinsicality at an instant supports my proposal to be perdurantist without being pointilliste - and so to block the RDA.

4.2.3 A better reason for temporal intrinsicality Finally, an incidental point. Philosophers discussing persistence have another reason to focus on temporally intrinsic properties, in addition to the erroneous tendency to think observational properties are temporally intrinsic to an instant. I admit that it is a better reason. But it is a reason only for properties temporally intrinsic for shortish intervals, up to about a second: not for the stronger notion of temporal intrinsicality at an instant - which is the target of my anti-pointilliste campaign. In short, the reason is that a property that is temporally intrinsic for a longish interval is liable to be useless in a criterion of identity.

In detail: All parties (both endurantists and perdurantists) can agree that an account of persistence, or a criterion of identity, had better not invoke a property that requires some feature of its instance within a period of time similar to the time-scale over which the account or criterion is to be applied. For doing so is liable to make the criterion hard or even impossible to apply. Thus suppose an account of the conditions under which $o$ at time $t$ is the same persisting object (maybe of a specific kind, e.g. person) as $o^{\prime}$ at $t^{\prime}$, invokes a property $P$ : requiring, say, that $o$ at $t$ must be $P$ and so must $o^{\prime}$ at $t^{\prime}$. (The argument works equally well with other requirements, e.g. that only one of the two need be $P$, but that change as regards $P$ is suitably continuous, with some kind of chain of small changes.) Then if being $P$ at $t$ requires a feature $\phi$ at a time close to $t^{\prime}$, it may well be hard to apply the account: having to ascertain that $\phi$ holds close to $t^{\prime}$ might entangle one in ascertaining whether the persistence claim for $o$ and $o^{\prime}$ holds.

\subsection{Non-instantaneous parts can do the jobs}

I turn to the second stage of my defence of perdurantism without instantaneous temporal parts. I claim that, by and large, non-instantaneous temporal parts do the various jobs, within the endurantism-perdurantism debate, that the perdurantist demands of temporal parts, just as well as instantaneous temporal parts. More precisely: this is so once the perdurantist "just says No" to the siren-calls of pointillisme. Of course, I cannot here discuss all these jobs: I will make do with three comments. The first comment is general, and will be illustrated by the second and third, which concern particular jobs temporal parts are invoked to do.

\footnotetext{
${ }^{16}$ Philosophers tend to forget that they also have trouble in physics. The classical mechanics and electrodynamics of point-particles and continua have considerable conceptual tensions, some of which are aggravated by a pointilliste picture; cf. (2004, 2004b).
} 
4.3.1 Humean supervenience revisited The first comment is an offer of a peacepipe to the neo-Humean. She envisages the world as "loose and separate", a succession of "distinct existences": "just one darned thing after another". My version of perdurantism can agree, in that it might well accept all non-instantaneous temporal parts, no matter how short-lived: my veto is only against utterly instantaneous parts.

Besides, my perdurantist can echo Lewis' Humean supervenience, by making some claim along the lines that all the facts supervene on the temporally local facts; i.e. the facts specified by the intrinsic (if you like: temporally and spatially intrinsic) properties of all the non-instantaneous temporal parts. To state this echo more precisely: she can claim that for any covering of spacetime $\mathcal{M}$ by a family $\mathcal{F}$ of non-instantaneous temporal parts (no matter how short-lived some or all of the parts may be), all the facts supervene on the intrinsic properties of elements of $\mathcal{F}$. (Here, 'covering' is understood in mathematicians' usual sense: a set $\mathcal{M}$ is covered by a family $\mathcal{F}$ of sets iff $\mathcal{M} \subseteq \cup \mathcal{F}$; and similarly if $\mathcal{M}$ and the elements of $\mathcal{F}$ are treated not as sets, but as say mereological fusions.)

So the only aspect of Lewis' Humean supervenience that my perdurantist needs to deny is the pointilliste idea that all the facts supervene on the intrinsic properties of spacetime points (or of spatially extended instants of time, i.e. spacelike surfaces). I think neo-Humeans should find this a price worth paying: having all the facts supervene on the intrinsic properties of all the non-instantaneous temporal parts should be enough to satisfy a Humean's ambition to have the "global" supervene on the "local". ${ }^{17}$

4.3.2 The problem of change The second comment concerns the so-called 'problem of change'. Perdurantists argue that $o$ 's changing in respect of a property $P$ is best understood in terms of one temporal part having $P$ and another having $\neg P$. In particular, they argue that the endurantist has to understand $P$ (and $\neg P$ ) as a relation to a time, and that for the case of an intrinsic property $P$ this is surely wrong. Hence the problem is also called the 'problem of temporary intrinsics'; (cf. e.g. Sider 2001 pp. 92-98, Lewis 2002).

So far as I can tell, almost all the arguments for the perdurantist understanding of change carry over, so as to support my version, i.e. perdurantism without instantaneous temporal parts. (Admitted: as do almost all the arguments against the perdurantist understanding of change.) The main reason is of course that if within a single non-instantaneous part of $o$ there is change in respect of $P$, the perdurantist will understand the change in terms of one shorter-lived part of $o$ having $P$, and another not-and this need not involve a vicious regress (Section 4.2.1). Besides: since temporal intrinsicality at an instant is rare (Section 4.2.2), the perdurantist's argument that endurantism has trouble with temporary intrinsics is more persuasive as an argument

\footnotetext{
${ }^{17}$ Agreed: since these parts in general overlap, the "fundamental description of the world", given by the infinite conjunction of all (the ascriptions of) the intrinsic properties of all such parts, is highly redundant. But I say: no worries. After all, the exact spatial analogue occurs in continuum classical mechanics. To describe a continuum, this theory needs - not the infinite point-by-point conjunction of all the properties of points-but the highly redundant infinite region-by-region conjunction of all properties of all regions; cf. footnotes 15 and 16.
} 
for non-instantaneous temporal parts.

But there is one objection; (my thanks to Oliver Pooley). Suppose that a temporary intrinsic property such as shape changes continuously over time, so that an object $o$ is square for merely an instant: to secure an instance of squareness simpliciter in this scenario, the perdurantist surely needs an instantaneous temporal part.

Reply: Given the supposition, this is certainly right. Here I can only bite the bullet, by any or all of:

(i) dropping the problem of change from the list of jobs my non-instantaneous temporal parts are to do; or

(ii) urging that since temporal intrinsicality at an instant is rare (Section 4.2.2) my temporal parts can solve the problem of change for the vast majority of temporary intrinsic properties; and besides, urging that succeeding with this vast majority should satisfy the neo-Humean (cf. the first comment above); or

(iii) adopting a "mixed" view, more congenial to pointillisme, that admits instantaneous parts as well as non-instantaneous ones, but then argues that it is legitimate to account for persistence (and so answer the RDA) by invoking only the non-instantaneous ones, as I have.

Of these options, I on the whole prefer reply (ii). But I will not in this paper try to choose between these replies: in particular, I will not refer again to the mixed view, though I agree it is tenable.

4.3.3 Puzzles of coincidence Thirdly, the situation as regards the debate over 'puzzles of coincidence' is similar to that for the problem of change. The puzzles (reviewed by Sider 2001, p. 5-10, 141-152) concern such cases as the statue and the clay, or the fission and fusion of objects such as amoebae - or even persons. For example, after an artist makes on Tuesday a statue out of a lump of clay, the statue and clay seem to be the very same object. But they seem to differ in their temporally extrinsic properties (often in this debate called 'historical properties', e.g. by Sider 2001, p. 5, 142): the statue but not the lump was created on Tuesday, the lump but not the statue existed on Monday. Perdurantists argue that these puzzles are best understood in terms of distinct objects sharing temporal parts, just as objects can share spatial parts (such as two roads having a stretch in common).

Again: so far as I can tell, almost all the arguments for the perdurantist understanding of these puzzles carry over, so as to support my perdurantism without instantaneous temporal parts. (As do, I admit, the arguments against!). For example, almost all the arguments in Sider's critique of endurantist approaches (2001, p. 154-188), and in his advocacy of perdurantism (2001, p. 152-153, p. 188-208), carry over.

I said 'almost all the arguments' carry over. For there are two wrinkles. First, Pooley puts the analogue of his objection in Section 4.3.2. Suppose that two objects fuse for merely an instant: here the perdurantist surely needs an instantaneous temporal part. I reply: I think this objection is weaker than its analogue in Section 4.3.2, because its supposition is more of an idealization, more a merely logical or metaphysical possibility, rather than part of the content of classical mechanics. That is: classi- 
cal mechanics does describe deformable objects changing shape continuously, as the objection in Section 4.3.2 requires. But it does not describe instantaneous fusions. Indeed, classical mechanics finds collisions, even of point-particles, problematic-let alone fusions and fissions. (There is of course no problem about the spatial analogue of instantaneous fusions, i.e. two 3-dimensional objects sharing a 2-dimensional part: think of two semi-detached houses!)

The second wrinkle is that the issue whether to accept instantaneous temporal parts does bear on one significant division within the perdurantist camp. This distinction concerns how the perdurantist treats temporal language. The traditional perdurantist view is that an object of ordinary ontology-i.e. a referent of an ordinary term, a subject of ordinary predications, an element of ordinary domains of quantification - is the whole four-dimensional object, the "maximal spacetime worm"; (Sider calls this the 'worm view'). But both Sider (2001, p. 188-208) and Hawley (2001, pp. 30-32, 41-64) defend the rival 'stage view', that the referents of our ordinary terms, subjects of ordinary predications etc. are the temporal parts.

This is not the place to assess their arguments for this proposal. They concern, for example, counting: the stage view says that at each time before an amoeba splits into two, there is one amoeba (the stage), a verdict which matches everyday thought and language; but since there are then two maximal spacetime worms, the worm view has to say that there are stricto sensu two amoebae, and explain away everyday thought and language by invoking some conventions about counting. ${ }^{18}$

For my purposes here, it suffices to comment on Sider's position that the stages he claims to be the referents of ordinary terms are indeed instantaneous - and so do not persist: 'no person lasts more than an instant' (2001, p. 193)! Sider of course agrees that everyday thought and language take: (i) ordinary objects to persist, as in 'Ted was once a boy'; and (ii) most of their properties to be temporally extrinsic at an instant, as in 'Ted believes perdurantism is true' (Section 4.2.2). So he goes on to argue that he can accommodate (i) and (ii) with a temporal analogue of Lewis' counterpart theory (2001, pp 111-113, 193-198).

My comment on Sider's position is now obvious. While I admit that temporal counterpart theory is coherent and powerful enough to cope with (i) and (ii) - the stage view does not have to be so pointilliste as Sider! That is: one could combine my perdurantism, the rejection of instantaneous temporal parts, with the stage view. Not only do most arguments for a perdurantist understanding of the puzzles of coincidence carry over and support my perdurantism (as I said above). Also, one could combine it with some arguments specifically for the stage view: e.g. a version of my perdurantism that denies overlapping parts could retain Sider's counting argument for favouring the stage view over the worm view ... But I leave developing this topic for another occasion.

\footnotetext{
${ }^{18}$ For this line of argument, cf. Sider 2001, pp 152-153, 188-193. But Sider has to admit that sometimes we count by maximal spacetime worms, not by stages, as in 'Fewer than two trillion people have set foot in North America throughout history'. He writes (2001, p. 197): 'if 'person' refers to person stages, this sentence will turn out false, since more than two trillion (indeed, infinitely many if time is dense) person stages have set foot in North America throughout history'.
} 
This concludes my metaphysical defence of this version of perdurantism. I hope to have made it plausible, quite apart from its blocking the RDA. But the philosophy of physics has some more support to offer it. In the next Subsection, the support comes from the classical description of motion. In Section 5, the support comes from quantum theory. But this support is not "just technical": each of them involves a novel proposal about the intrinsic-extrinsic distinction among properties.

\subsection{Instantaneous velocity is hardly extrinsic}

Extrinsicality is usually considered an all-or-nothing affair. But it is natural to suggest that it comes in degrees. Intuitively, a property is more extrinsic, the more that its ascription implies about the world beyond the property's instance: (compare the philosophy of mind's jargon of 'wide' and 'narrow' mental states - some are wider than others). ${ }^{19}$ That is rough speaking; and all the rougher because of controversies about the intrinsic-extrinsic distinction. But I expect that in many sufficiently limited contexts, the idea could be made precise in a natural way. In any case, I shall in this Subsection only consider the temporal extrinsicality at an instant of the properties of position and its time-derivatives (velocity, acceleration etc.), in the classical description of motion. This is certainly a sufficiently limited context for the idea to be made precise. After making it precise, I will return to perdurantism without tears, i.e. without instantaneous temporal parts. ${ }^{20}$

In (1) and (2) below, I present two closely related ways of making it precise. On both ways, instantaneous velocity is "hardly extrinsic", i.e. hardly temporally extrinsic, since its ascription to an object $o$ at $t$ implies "little" about matters of fact at other times. ${ }^{21}$ Both ways are based on the obvious point that the only "categorical" proposition that an ascription of a velocity (or indeed, of a higher derivative of position) to $o$ at $t$ implies about other times is that the $o$ exists for some open interval $(a, b)$ containing $t$ : all the other implications are hypothetical. This will support my perdurantism without instantaneous temporal parts.

(The difference between the two ways will be that according to the first, which is

\footnotetext{
${ }^{19}$ It is all the more natural when we consider how few properties are intrinsic: the extrinsic properties form so large a class as to merit being sub-divided.

${ }^{20}$ Here I should stress a limitation of my discussion, which I have imposed since Section 2.2 and will maintain until Section 5.1. Namely: in order to give the RDA as good a run as possible, I go along with the RDA literature's assumption that velocity is defined as the time-derivative of position (so that position is conceptually prior to velocity, and momentum is defined as mass times velocity). But this assumption is questionable, for various reasons. In particular, one can develop classical mechanics by taking momentum as primitive, together with position and mass, and defining velocity as momentum divided by mass. And in such a presentation, momentum does not need to be "secretly understood" as mass times velocity: one can introduce it abstractly, and without reference to time, as the generator of spatial translations. Thanks to Gerard Emch for explaining this point.

${ }^{21}$ It is also hardly temporally extrinsic, on a third construal of that notion discussed in my 2004. Of course none of this is to deny that instantaneous velocity is temporally extrinsic at an instant, since it presupposes persistence.
} 
"read off" the calculus, successively higher time-derivatives of position are more extrinsic; while on the second way, which is more logical and less mathematical, velocity acceleration and all higher derivatives are equally - and only mildly - extrinsic.)

In what follows, we can think of $o$ as a point-particle; but it could equally well be a point-sized piece of matter in a continuum, or an extended body small and rigid enough to be treated as a point-particle. It will also be clear that the temporal extrinsicality of average velocity, acceleration etc. is mild for essentially the same reasons as for instantaneous velocity, acceleration etc. But to save space, I will focus on the instantaneous quantities.

(1): The sequence of time-derivatives: - The discussion will be tidier if we consider ascriptions, not of specific values of position, velocity, acceleration etc. to $o$ at time $t$, but of some or other value. Then successive ascriptions are of increasing logical strength: having a velocity implies having a position, having an acceleration implies having a velocity etc.

So consider a sequence of ascriptions to $o$ at time $t$ : viz.

(Pos): an ascription of a position, i.e. a proposition saying that $o$ has some or other position at $t$;

(Vel): an ascription of an (i.e. some or other) instantaneous velocity at $t$;

(Acc): an ascription of an instantaneous acceleration at $t$.

These ascriptions are of course the first three members of an infinite sequence of ascriptions stating the existence of higher time-derivatives of $o$ 's position. This gives an obvious sense in which instantaneous velocity is only mildly extrinsic. Each ascription is logically stronger than its predecessor; so (Vel), being almost at the start of the sequence, implies little in comparison with later members.

In more detail: if a real function $f$ has a derivative at a point $t \in \mathbb{R}$, it must be defined on a neighbourhood of $t$ and be continuous at $t$. So the existence of $f^{\prime \prime}(t)$ requires the existence of $f^{\prime}$ in a neighbourhood of $t$ and its (i.e. $f^{\prime \prime}$ 's) continuity at $t$; and this in turn requires the continuity of $f$ in that same neighbourhood of $t$. And so on. In short: the existence of the $n$th derivative gives more information about times other than $t$ than does the existence of the $(n-1)$ th derivative.

(2): The "only categorical implication":- But there is also another sense in which velocity and the higher derivatives of position are only mildly temporally extrinsic. This sense is more directly tied to the basic idea that the only categorical proposition that an ascription of such a quantity to $o$ at $t$ implies about other times is that the $o$ exists for some open interval $(a, b)$ containing $t$.

In more detail. Let us ask what exactly is implied about other times by the ascriptions in the sequence; starting with (Pos). The metaphysical literature invariably assumes position to be temporally intrinsic: why? The answer seems clear: 'because (Pos), or even an ascription of a specific value ' $o$ is at $\mathbf{x}$ at $t$ ', implies nothing about $o$ 's position at other times'. ${ }^{22}$

\footnotetext{
${ }^{22}$ Once again, as mentioned at the end of Section 2.1: this answer ignores possible implications about other objects' positions, at $t$ or other times, and so sets aside the absolute-relational debate about space.
} 
But to be more precise about 'implying nothing' (apart of course from necessary or analytic propositions), we need:

(a) to decide whether to allow that the object $o$ might exist only for an instant; (as many metaphysical discussions of persistence do: true to the tradition of conceptual analysis, they allow all metaphysical or logical possibilities, not just the nomic ones); and

(b) to distinguish categorical from hypothetical propositions.

Although the categorical-hypothetical distinction is vague and contentious (because 'logical form' is), I will not need to be precise or partisan about this: for it will be obvious from the calculus' definition of a limit which propositions implied by ascriptions such as (Pos)-(Acc) to count as hypothetical.

If we allow $o$ to exist only for an instant (if we say 'Yes' in (a)), then indeed (Pos) implies no categorical proposition about $o$ 's positions at other times: there may be no such positions! But consider a hypothetical proposition along the lines: 'if $o$ exists at a later time $t^{\prime}$, and some value (or upper limit) is assumed about its average speed (defined in the usual way as distance traversed divided by time elapsed) over $\left[t, t^{\prime}\right]$, then $o$ is at $t^{\prime}$ within a sphere of a certain radius, centred on $\mathbf{x}^{\prime}$. Such a hypothetical proposition is of course not analytic; but it follows by just definitions and logic from ' $O$ is at $\mathbf{x}$ at $t$ '.

When we turn to the next member of the sequence, (Vel), we of course get many more implications. o must exist throughout some open interval, maybe tiny, around $t$; and since differentiability implies continuity, o's position at a time $t^{\prime}$ in the interval tends, as $t^{\prime}$ tends to $t$, to $o$ 's position $\mathbf{x}$ at $t$; and so on. But these implied propositions are, with one exception, hypothetical. The hypothetical propositions include those about average velocity discussed in the previous paragraph, and various others one can spell out by applying the definitions of continuity and differentiability. The exception is of course the categorical proposition that $o$ exists throughout some open interval of times around $t$; (and, to be precise: its analytic consequences, like $o$ 's existing at some time $t^{\prime}$ not equal to $t$ ). In particular, (Vel) is compatible with $o$ being anywhere at any other time $t^{\prime}$, no matter how close $t^{\prime}$ is to $t .^{23}$

Similarly again, for (Acc). There are again more implications, but they are almost all complicated hypotheticals: the only categorical proposition about other times that the ascription implies is the same one again: that $o$ exists throughout some open interval of times around $t$. And so on along the infinite sequence of ascriptions.

To sum up this discussion of (1) and (2): the temporal extrinsicality of velocity and higher derivatives of position is mild. For almost all of the implied propositions are hypothetical; and even the temporally intrinsic ascription (Pos) implies countless such propositions. Besides, the categorical propositions implied by an ascription of velocity, or of any higher derivative, are all just consequences of the one proposition that $o$ exists throughout some open interval of times around $t$. So as regards categorical implications about other times, the temporal extrinsicality gets already at stage (Vel)

\footnotetext{
${ }^{23}$ I here assume there is no limiting velocity, as in relativity.
} 
as "bad" as it ever gets along the sequence: and that, I submit, hardly deserves the name 'bad' - it is mild. ${ }^{24}$

Finally, I can connect this discussion with the previous Subsections' advocacy of a perdurantism accepting only non-instantaneous temporal parts. The main idea here has been that the "only proposition that goes beyond the instance" implied by an ascription of velocity (or of other derivatives of position) is that $o$ exists throughout some open interval, perhaps very short, around the time $t$. So the "only proposition that goes beyond the instance" that velocity (or other derivatives of position) implies, corresponds to an intrinsic property of any (no matter how short!) non-instantaneous temporal part containing $t$.

Obviously, this idea meshes with two points in previous Subsections:-

(a): A perdurantist who rejects instantaneous temporal parts can account for persistence: either by fusing segments, perhaps arbitrarily short, of worldlines ("kinematics"; Section 4.1.1); or (more naturalistically) by "piggy-backing" on solving the deterministic classical laws of motion, given o's initial position and velocity, and the forces on it; ("dynamics"; Section 4.1.2).

(b): A perdurantism without instantaneous temporal parts can accept all noninstantaneous parts, no matter how small their temporal extent. Besides, if one accepts all such parts one can add a claim that all facts supervene on the "temporally local" facts, in a strong enough sense to satisfy all but the most pointilliste neo-Humeans; (cf. Section 4.3.1).

\section{Support from decoherence in quantum theory}

\subsection{Classical and quantum: relativizing the intrinsic-extrinsic distinction}

As I said in Section 2.3, I am not so far gone in naturalism as to just dismiss the RDA on the grounds that matter is in fact atomic. I agree: a classical mechanical continuum could exist-prompting the RDA, modulo the above replies. My argument in this Section will instead be that the way in which classical mechanical objects (both particles and continua) are in fact emergent from the quantum realm provides further support for Section 4's perdurantism without tears, i.e. without instantaneous temporal parts.

This argument will use two new assumptions: one about philosophical method, the other about the intrinsic-extrinsic distinction.

\footnotetext{
${ }^{24}$ This view is reflected in the jargon of mathematics and physics. For example, mathematicians call not only (Pos), but also the ascriptions (Vel) etc., 'local'; and physicists call equations of motion that determine the object's motion at $t$ in terms of its position and some of its derivatives then (but without reference to facts a finite temporal interval from $t$ ) 'local in time'. For more discussion, cf. Arntzenius 2000 pp. 192-195, Smith 2003 and my 2004b.
} 
(1): I will now assume that the interpretation of classical mechanics - in particular, our conception of how its objects (both particles and continua) persist - should be sensitive to how classical mechanical objects in fact "emerge from the quantum". I agree that this assumption is controversial: why not just interpret each theory on its own, as best you can? After all, there is no lack of work: as I remarked in Section 2.3, classical mechanics is interpretatively subtle and problematic, even without considering the dreaded quantum. But I am not alone in endorsing this assumption, even as regards the interpretation of a classical theory being sensitive to an "adjacent" quantum theory. Thus for Belot (1998, p. 550-554), it is the main moral of his examination of classical electromagnetism and the Aharonov-Bohm effect.

(2): My second assumption is that it is legitimate to relativize the intrinsic-extrinsic distinction among properties to a body of doctrine. The distinction is of course usually discussed in terms of logical or metaphysical possibility: the literature discusses taking a property $P$ to be intrinsic iff it is logically or metaphysically possible for an object $o$ to have $P$ "while lonely", or "whatever the rest of the world is like", or ... But I now assume that it is legitimate to relativize the modality to a body of doctrine, such as a scientific theory $T$. (I will not need the metaphysically more ambitious idea of relativizing to the "laws of nature", or to the laws of nature of some possible world.) Therefore I shall talk, for any such body of doctrine or theory $T$, of nomic intrinsicality and extrinsicality.

Unless $T$ is logically or metaphysically necessary - a case I need not consider - the relativized modality will be a restricted one. That is: not all logically or metaphysically possible worlds make $T$ true. In general, this will strengthen the notion of intrinsicality, and correspondingly weaken the notion of extrinsicality-however exactly we understand the original intrinsic-extrinsic distinction. That is: nomic intrinsicality will imply intrinsicality simpliciter, and extrinsicality simpliciter will imply nomic extrinsicality. For intrinsicality is a matter of "not implying propositions about the instance's environment"; and once we assume a theory $T$ is true, any proposition in $T$ can be an implicit premise in an implication-yielding more implications. So in general, once we assume $T$, more properties will be classified as extrinsic. So extrinsicality simpliciter implies nomic extrinsicality; and vice versa for intrinsicality. (Similar remarks apply to my notions of temporal, and spatial, intrinsicality and extrinsicality; and to the case where we consider two theories $T_{1}$ and $T_{2}$, one implying the other.)

In fact, this idea of relativized intrinsicality has surfaced in the literature (Humberstone 1996, p. 238); but so far as I know, it has not been pursued. I agree that many a metaphysician will at first sight doubt its value, though they will probably accept it as coherent. Thus Humberstone writes, after floating the idea of relativizing intrinsicality to a class of possible worlds that match in their laws of nature: 'From a suitably elevated position [i.e. suitably general philosophical stance], this has an element of arbitrariness about it: why not restrict attention to worlds - not with the same laws as ours, but - with the same tourism statistics for Naples as ours?' (ibid.).

But I submit that relativization to (our best guess for) the laws of physics has some interest! In any case, I can at least show that in the present context, it has the interest 
of being surprising. For in Section 5.2 I will argue that the position, and even the existence, at a time of an emergent classical object (whether a particle or a point-sized piece of matter in a continuum) is extrinsic, relative to the laws of quantum theory. ${ }^{25}$

But before arguing for this, I should briefly set aside another way in which quantum theory bears on persistence, and apparently on the RDA.

\subsubsection{Unitarity: momentum as temporally intrinsic}

Quantum theory violates an assumption that the RDA depends on, viz. that velocity is not part of the instantaneous state of an object (in particular a point-sized piece of matter). (I registered this assumption already in Section 2.2; and set aside Tooley's heterodox proposal that velocity should be thus viewed, on the grounds that Tooley's proposal in any case assumed the notion of persistence.) This assumption is often endorsed in the metaphysical literature about space, time and motion, even apart from the RDA: for example, Sider (2001, p. 39) says 'fixing the properties and relations of present objects will not fix their velocities' (cf. also his p. 34-35).

The assumption tends to be associated with the fact that in classical mechanics, in order to determine an object's future (and past) motion, you need not only its present position and the forces acting on it (in the time-interval concerned), but also its present velocity; i.e. the fact that classical mechanics' equations of motion are second-order in time. For in a theory in which position and forces were enough to determine the motion (a theory that is first-order in time), it would be more tempting to say that velocity is part of the present instantaneous state. At least, it would be as tempting to say this, as that the whole future (and past) history of the system is part of the present instantaneous state (because of the determinism). Certainly, in such a theory the RDA itself would have much less sting for a "naturalistic" perdurantist, who is willing to let her account of perdurance depend on the actual laws. For in such a case, ingredients that the RDA's advocate presumably agrees to be available to the perdurantist, viz. position and forces, are enough to determine future positions.

But quantum theory violates this assumption. ${ }^{26}$ It is first-order in time. It combines the position and velocity (better: momentum) aspects into a single instantaneous state of a system which, together with the forces acting on the system, determines its future (and past) states (setting aside controversy about whether there is a "collapse of the wave-packet" on measurement). ${ }^{27}$

\footnotetext{
${ }^{25}$ Besides, the extrinsicality has nothing to do with the possible involvement of other objects in defining position, as urged by a relational conception of space: an issue I set aside at the end of Section 2.1. The extrinsicality is what I have called temporal extrinsicality, rather than spatial; and it arises from decoherence.

${ }^{26}$ As readers who are cognoscenti of quantum theory will have long ago noticed: at least by the time that Section 4.1 proposed we could have perdurantism without tears, by letting the perdurantist "have" velocity, and even have their account of persistence "piggy-back" on integrating the classical equations of motion. Apologies for the delay!

${ }^{27}$ Agreed, the Hamiltonian formulation of classical mechanics also combines position and momentum
} 
So it is tempting to say that in quantum theory, velocity and momentum are just as intrinsic (or temporally intrinsic) to the system at a time, as is position; (Arntzenius (2003, p. 282) says this). A bit more precisely: once we are willing to relativize the intrinsic-extrinsic distinction to a physical theory (as proposed in (2) above), it is tempting to say this.

Furthermore, just as Section 4.1.2 proposed that in a classical setting, a perdurantist accepting only non-instantaneous parts could have their account of persistence "piggy-back" on integrating the classical equations of motion: so in quantum theory, the perdurantist's account of persistence could appeal to integrating the quantum equations of motion. (But as the weasel-word 'system' hints, it is controversial how to relate persisting objects to quantum systems, even if you know the systems' complete histories: cf. the next Subsection).

So be it, say I. But again: I am not so far gone in naturalism about persistence-I am loath to just dismiss the RDA on the grounds that quantum theory is first-order in time. A theory of persistence should accommodate classical continua, and this Subsection's points do not bear directly on how it can do so. However, I will now argue that quantum theory has other light to shed on our topic-once we ask the interpretation of classical mechanics to take note of how classical mechanical objects emerge through decoherence.

\subsection{Position and existence as nomically extrinsic}

So let us adopt the idea in (2) of Section 5.1, of nomic intrinsicality and extrinsicality. The intrinsic-extrinsic distinction among properties is to be relativized to bodies of doctrine - in particular, to quantum theory.

Warning: Choosing logically strong bodies of doctrine can yield odd-sounding verdicts of extrinsicality. Given our interest in temporal extrinsicality, the obvious example of this is provided by a deterministic theory. Thus suppose you choose to relativize, not just to the deterministic theory itself, but to the conjunction of the theory and the regime of forces imposed on a system in some time-interval $(a, b)$. This yields the verdict that every instantaneous state ${ }^{28}$ is temporally very extrinsic: indeed, about as extrinsic as it could be. For given the laws of the theory and the forces imposed, any instantaneous state of a system determines the system's states during $(a, b)$. But it sounds wrong to say that every instantaneous state is temporally very extrinsic.

The solution of course is to exercise some judgment about what is a natural or useful body of doctrine to which to relativize. In our example, the theory is presumably

in its conception of state, and so is first-order in time. But there is a crucial disanalogy: neither of the pair, position and momentum, determines the other. (Indeed, the formulation is equivalent to the Lagrangian or Newtonian formulation, under certain conditions, in particular taking the phase space to be the cotangent bundle of a configuration space.) But in quantum theory, the position and momentum representations each determine the other.

${ }^{28}$ Since for some philosophers, a state is not a property, it is better to say: every property that specifies such a state. 
such a body of doctrine, but its conjunction with a specified regime of forces is not: that is too particular (logically strong). More generally, we should allow some distinction between "central" and "peripheral" statements (or more generally; features) of an ambient body of doctrine, and relativize the intrinsic-extrinsic distinction only to (the conjunction of) the central ones. That is, only the central ones are held fixed in all the nomic possibilities, and so by nomic intrinsicality and extrinsicality. Then you may say in the example that (maybe part of) the specification of the forces is not central, so that instantaneous states are not so very temporally extrinsic.

Let us now apply this sort of relativization to how classical mechanical objects emerge through decoherence. Fortunately for us, although the quantum measurement problem remains controversial and there remain many open technical questions in the physics of decoherence, we need not address these controversies and questions. We can sidestep the measurement problem, and manage with only the most basic and best-established features of decoherence. ${ }^{29}$

Classical mechanical objects (both particles and continua) are in fact transient and approximate patterns in the quantum state of an underlying quantum system. They are patterns that emerge from an ubiquitous, continuous and very efficient process of decoherence, which continues throughout the lifetime of the classical object. Roughly speaking, decoherence is diffusion (spreading) in to the quantum system's environment of coherence, i.e. of the puzzling interference effects in the probability distributions that are the system's state.

To keep things simple, I shall discuss this in terms of the elementary quantum theory of particles, not quantum field theory. But I should note that:

(i): quantum particles are themselves transient and approximate patterns in the quantum state of an underlying quantum field or fields; for discussion of this, cf. my (2004b) and Wallace (2004, especially Section 5.2);

(ii): decoherence also happens within quantum field theory; for a review, cf. Guilini et al. (2003, Chapter 4).

Here are some details about a well-studied model of a quantum particle immersed in an environment (called 'quantum Brownian motion'). Take as the initial quantum state of a tiny dust-particle (radius $10^{-3} \mathrm{~cm}$ ) in air, a superposition of two positions for the centre of mass of the particle, with the two positions just $10^{-4} \mathrm{~cm}$ apart (i.e. a tenth of the particle's radius), and with (say) the two positions not moving relative to one another. The bombardment of the particle by air molecules is very efficient in diffusing the coherence in to the environment: the superposition's interference effects converge to zero like $\exp \left(t / 10^{-36} \mathrm{sec}\right)$ and remain small for a very long time $\left(10^{10}\right.$ years)!

This means that very soon the probabilities for any quantity on the particle you care to measure are as if there is an even chance of the (centre of mass of the) particle

\footnotetext{
${ }^{29}$ Bacciagaluppi (2003) is an excellent introduction to decoherence for philosophers; for more technical details, Guilini et al. (2003), Schlosshauer (2003) are also excellent. By the way, all these sources endorse the consensus that decoherence cannot by itself provide the solution of the measurement problem, but is an important ingredient in any such solution.
} 
being in the two positions; (i.e. probabilities for quantities other than position are also given by a 50-50 mixture corresponding to the two positions). Similarly for other initial states: if the initial superposition had the two positions separating from each other at say $x \mathrm{~cm} \mathrm{sec}^{-1}$, then after a second, the probabilities would be as if there is an even chance of the (centre of mass of the) particle being in two positions $x+10^{-4} \mathrm{~cm}$ apart. Indeed, more generally: it is even possible to deduce the approximate validity of the deterministic classical mechanical equations of motion of a dust-particle from the underlying equations for the quantum system, together with a description of the decoherence process.

So the classical object, "the dust-particle we see", corresponds to one of these two decohered possibilities (in my example: possibilities for the position of the centre of mass). It is a pattern in a quantum state, which also contains another pattern corresponding to the other possibility. If the quantum state were sufficiently different, not only would the classical object not have the position and momentum we see: it would not exist. In particular, if the decoherence process did not occur, it would never exist; and if the decoherence process did not continue, it would cease to exist. That is: the quantum system would continue to exist, but the classical dust-particle would not: it would "disappear into a quantum fog". ${ }^{30}$

I propose that we take these points, about how classical objects are in fact patterns in a quantum state that are formed because of an ongoing process of decoherence, as what I called 'central'. After all, they are crucial to how such objects are in fact constituted. That is: I propose they are to be held fixed in assessing whether a property is nomically intrinsic or extrinsic. So they are to be available as implicit premises for implications from ascriptions of a property to propositions about the world beyond the property's instance.

It follows that an ascription to a classical object such as a dust-particle, of a position at $t$ (to be precise: for its centre of mass, say), is nomically extrinsic. (I would say: temporally extrinsic, since the implications are about facts at times other than $t$ ). For the ascription (together with the implicit premises) implies the (categorical) proposition that the object has a position at all other times in a (very short but non-zero!) interval of times around $t$. Here, the length of the interval is determined by the decoherence process' time-scale.

Similarly, a statement that the object exists at a time is nomically extrinsic. For it implies that the object exists at all other times in an interval about as long as the decoherence time-scale. ${ }^{31}$

\footnotetext{
${ }^{30}$ For more discussion of the idea of classical objects as patterns in quantum objects, cf. Wallace (2003).

${ }^{31} \mathrm{~A}$ point of clarification for quantum aficionados. You might object that since

(i) the reduced state density matrix of the dust-particle (strictly: of its centre of mass degree of freedom) is nearly diagonal (upto some desired level of approximation) in position, at an instant; it surely follows that:

(ii) the position and existence of the classical particle is not nomically temporally extrinsic.

I reply: (i) is of course true, but does not imply (ii). For I am taking as "central", not just the formalism of reduced states etc., but also the physical fact of a decoherence process over time.
} 
So far I have only discussed the emergence of a classical particle, such as a dustparticle. But the discussion just given carries over to continua, as regards both physics and philosophy.

Admittedly, there are more technical questions about decoherence in quantum fluids that are still open than about quantum Brownian motion, which is by now very wellstudied. But there is already a good understanding of decoherence in quantum fluids, and so of the emergence of classical continua. In short: recent work shows that even in a quantum fluid, where there is no clear distinction between system and environment, decoherence selects certain quantities (roughly, hydrodynamic variables) as "behaving classically". Again, one can deduce the approximate validity of the classical equations of motion for a fluid. (For details, cf. Halliwell (1999) and references given there.)

As regards philosophy: I said above that the fact that classical mechanical objects (both particles and continua) are in fact emergent from the quantum realm should be reflected in the interpretation of classical mechanics, and so in a naturalistic theory of persistence. One way to do this is now clear. Namely: take the nomic extrinsicality of position at a time, and even existence at a time, as favouring the denial of instantaneous temporal parts. Thus decoherence supports the perdurantism without the tears of pointillisme which I defended in Section 4: a naturalistic perdurantist can interpret classical mechanics in terms of temporally extended temporal parts - and thereby block the RDA.

Acknowledgements:- I thank: G. Belot, C. Callender, W. Demopoulos, G. Emch, I. Gibson, L. Humberstone, P. Mainwood, A. Oliver, O. Pooley, M. Redhead, T. Sattig, S. Saunders, N. Shea, D.Wallace, A. Wilson and D. Zimmerman for discussions and comments on previous versions; audiences in Florence, Granada, Leeds, London England, London Ontario, Montreal and Oxford; and participants at a seminar in autumn 2003.

\section{References}

R. Abraham and J. Marsden (1978), Foundations of Mechanics, 2nd edition, Benjamin/Cummings.

D. Armstrong (1980), 'Identity through time', in ed. P. van Inwagen, Time and Cause, Dordrecht: Reidel.

D. Armstrong (1997), A World of States of Affairs, Cambridge: University Press.

F. Arntzenius (2000), 'Are there really instantaneous velocities?', The Monist 83, pp. 187-208.

F. Arntzenius (2003), 'An arbitrarily short reply to Sheldon Smith on instantaneous velocities', Studies in the History and Philosophy of Modern Physics 34B, pp. 281-282. G. Bacciagaluppi (2003), 'The role of decoherence in quantum theory', in ed. E. Zalta, The Stanford Encyclopedia of Philosophy, available at: http://plato.stanford.edu.

G. Belot (1998), 'Understanding electromagnetism', British Journal for the Philosophy of Science 49, pp 531-555. 
J. Bigelow and R. Pargetter (1989), 'Vectors and Change', British Journal for the Philosophy of Science 40, pp 289-306.

J. Bigelow and R. Pargetter (1990), Science and Necessity, Cambridge University Press.

J. Butterfield (2004), 'Classical mechanics is not pointilliste and can be perdurantist', in preparation.

J. Butterfield (2004a), 'On the persistence of particles', forthcoming in Foundations of Physics; available on the Los Alamos arXive at http://xxx.lanl.gov/abs/physics/0401112, and on the Pittsburgh philosophy of science arXive at: http://philsci-archive.pitt.edu/archive/000015 J. Butterfield (2004b), 'On the persistence of homogeneous matter', available on the Pittsburgh philosophy of science arXive at: http://philsci-archive.pitt.edu/archive/00001760/. C. Callender (2001), 'Humean supervenience and rotating homogeneous matter', Mind 110, pp. 25-42.

F. Diacu and P. Holmes (1996), Celestial Encounters: the Origins of Chaos and Stability, Princeton University Press.

K. Devlin (2002), The Millennium Problems, London: Granta Books.

W. Dixon (1978), Special Relativity, Cambridge: University Press.

J. Earman (1989), World Enough and Space-Time, Cambridge MA: MIT Press.

I. Grattan-Guinness (2002), 'Algebras, Projective Geometry, Mathematical Logic and Constructing the World: intersections in the philosophy of mathematics of A.N. Whitehead', Historia Mathematica 29, pp. 427-462.

D. Guilini et al. (2003), Decoherence and the Appearance of a Classical World in Quantum Theory, New York: Springer (2nd edition).

J. Halliwell (1999), 'The Emergence of Hydrodynamic Equations from Quantum Theory: A Decoherent Histories Analysis', to appear in Proceedings of the 4th Peyresq Conference, 1999; available at: http://xxx.soton.ac.uk/abs/quant-ph/9912037

K. Hawley (2001), How Things Persist, Oxford University Press.

I. Humberstone (1996), 'Intrinsic/Extrinsic, Synthese 108, pp. 205-267.

G. Leibniz (1698), 'On Nature itself, Or On the inherent force and actions of created things'; pp. 498-508 of L.E. Loemker ed. (1989) Gottfried Wilhelm Leibniz: Philosophical Papers and Letters, Dordrect: Kluwer.

D. Lewis (1986), Philosophical Papers, volume II, New York: Oxford University Press. D. Lewis (1994), 'Humean Supervenience Debugged', Mind 103, p 473-490; reprinted in his Papers in Metaphysics and Epistemology (1999), Cambridge University Press pp.224-247.

D Lewis (1999), 'Zimmerman and the Spinning sphere', Australasian Journal of Philosophy 77, pp. 209-212.

D Lewis (2002), 'Tensing the copula', Mind 111, pp. 1-13.

Malament (2002), 'A No-Go theorem about rotation in relativity theory', in D. Malament ed. Reading Natural Philosophy Chicago: Open Court; p. 267-293.

Malament (2003), 'On relative orbital rotation in relativity theory', in A. Ashtekar, R.Cohen et al. eds. Revisiting the Foundations of Relativistic Physics: Festchrift for John Stachel, Kluwer Academic; pp. 175-190.

C. Misner et al. (1973), Gravitation, San Francisco: Freeman.

D. Robinson (1989), 'Matter, Motion and Humean supervenience', Australasian Jour- 
nal of Philosophy 67, pp. 394-409.

M. Schlosshauer (2003), 'Decoherence, the measurement problem, and interpretations of quantum mechanics', available at the Los Alamos physics arXive: quant-ph/0312059. T. Sider (2001), Four-Dimensionalism, Oxford University Press.

S. Smith (2003), 'Are instantaneous velocities real and really instantaneous? An answer in the affirmative', Studies in the History and Philosophy of Modern Physics 34B, 261-280.

M. Tooley (1988), 'In defence of the existence of states of motion', Philosophical Topics 16, pp. 225-254.

D. Wallace (2003), 'Everett and structure', Studies in the History and Philosophy of Modern Physics 34B, 87-105.

D. Wallace (2004), 'In defence of naivete: The conceptual status of Lagrangian quantum field theory', forthcoming in Synthese;

available at: http://philsci-archive.pitt.edu/archive/00000519.

R. Wald (1984), General Relativity, Chicago: University Press.

B. Weatherson (2002), 'Intrinsic and extrinsic properties', in ed. E. Zalta, The Stanford Encyclopedia of Philosophy, available at: http://plato.stanford.edu.

D. Zimmerman (1998), 'Temporal parts and supervenient causation: the incompatibility of two Humean doctrines', Australasian Journal of Philosophy 76, pp. 265-288. 